Reprod. Nutr. Dévelop. 1980, 20 (4 A), 929-938.

\title{
Effets comparés d'un régime enrichi en yoghourt vivant ou thermisé sur le système immunitaire de la souris
}

\author{
par Gilles-Alice CONGE, Patricia GOUACHE, J. P. DESORMEAU-BEDOT *, \\ F. LOISILLIER **, D. LEMONNIER
}

Unité de Recherches sur la Nufrition ef l'Alimentation,

U. 1. de I'INSERM, Hôpital Bichat,

170 bd Ney, 75877 Paris Cedex 18.

* Institut Pasteur, 92380 Garches.

** Instifut Gustave Roussy, 94800 Villejuif.

Summary. Comparative effects of live yogurt and heated yogurt-enriched diets on the murine immunosystem.

Growth was fastest in mice fed yogurt. There was no difference in the effects of live or heated yogurt on the ponderal curve or the organ weight of vaccinated or unvaccinated mice. Sera antitoxin levels were slightly elevated in animals fed live yogurt and given an antitetanus vaccination at the particular concentrations used. Significantly high $\lg _{2} a$ immunoglobulin levels were found only in unvaccinated mice fed live yogurt. Histological examination before, and particularly after, antitetanus vaccination confirmed these findings in the thymus and especially in the spleen.

\section{Introduction.}

Certains effets sur l'hôte de l'ingestion de yoghourt qui contient des bacilles lactiques (Lactobacillus bulgaricus), des streptocoques (Streptococcus thermophilus) ont déjà été observés : Reddy ef al. (1973) a montré une action antitumorale du yoghourt chez la souris, attribuée à une substance antitumorale. On peut penser à d'autres mécanismes, en particulier un effet sur les défenses immunitaires. Dans ce but, on a déterminé des titres d'immunoglobulines sériques avant et après immunisation par un vaccin antitétanique et effectué les examens histologiques de la rate et du thymus de souris qui ont consommé des régimes enrichis en yoghourt présentant une flore vivante ou tuée.

\section{Matériel ef méthodes.}

150 souris Swiss femelles (CNRS, Orléans-La Source) de 4 semaines sont réparties par randomisation en cages de 5 animaux sur litière de sciure dans une animalerie de type conventionnel. La température est maintenue d̀ $22 \pm 1{ }^{\circ} \mathrm{C}$, l'éclairement au 
moins pendant $12 \mathrm{~h}$ sur 24 . Elles reçoivent pendant 15 semaines l'un des 3 régimes suivants, préparés au laboratoire et administrés ad libitum :

- régime témoin $T_{3}$, en poudre, à base de farine, caséine, son et enrichi en vitamines et minéraux (Lemonnier, 1972), non stérilisé. Il apporte 9 p. 100 de calories lipidiques, $22 \mathrm{p}$. 100 de calories protéiques, soit au total 340 calories pour $100 \mathrm{~g}$. Il est conservé à $+4{ }^{\circ} \mathrm{C}$;

- ce régime auquel on ajoute du yoghourt vivant ;

- ce régime auquel on ajoute du yoghourt thermisé.

Le yoghourt vivant ou thermisé est incorporé à froid au régime $T_{3}$, ce qui constitue une sorte de pâte qui est distribuée dans des mangeoires de porcelaine. Cette adjonction de yoghourt au régime $T_{3}$ est effectuée extemporanément tous les 2 jours, pour distribution immédiate.

Les yoghourts vivants utilisés contiennent $10^{7}$ à $10^{8}$ bacilles lactiques bulgares ef $10^{8}$ streptocoques thermophiles par gramme. Un test préliminaire de dénombrement in vitro des bacilles au moyen de la méthode classique du nombre le plus probable, selon Mac Crady (par ensemencement de dilutions successives sur milieu liquide au lait tournesolé), a été effectué au bout de $48 \mathrm{~h}$ dans différents mélanges de régime $\mathrm{T}_{3}$ et de yoghourts conservés à $20^{\circ} \mathrm{C}$ : on voit (tabl. 1) qu'un taux de 35 p. 100 en poids de

\section{TABLEAU $\uparrow$}

Dénombrement de bacilles vivants du yoghourt sur rations alimentaires contenant différentes concentrations en yoghourt et conservées $48 \mathrm{~h} d 4^{\circ}$ et $20^{\circ} \mathrm{C}$.

(Résultats en germes par grammes de ration)

Conservation à $4^{\circ} \mathrm{C}$

Conservation à $20^{\circ} \mathrm{C}$

Yoghourt 25 p. 100 non sucré ..........

Yoghourt 25 p. 100 sucré ...............

Yoghourt 35 p. 100 non sucré ............

Yoghourt 35 p. 100 sucré ..............

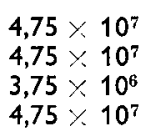

$4,75 \times 10^{7}$
$4,75 \times 10^{7}$

$3,75 \times 10^{6}$

$4,75 \times 10^{7}$

$7,5 \times 10^{5}$
$7,5 \times 10^{4}$
$2,25 \times 10^{7}$
$1,25 \times 10^{4}$

$7,5 \times 10^{5}$
$7,5 \times 10^{4}$

$2,25 \times 10^{7}$

$1,25 \times 10^{4}$

Technique utilisée : dénombrement sur milieu liquide (laił tournesolé).

Les chiffres correspondent au nombre de germes contenus dans la ration alimentaire (bacilles lactiques + streplocoques thermophiles).

yoghourt non sucré permef la meilleure survie des bacilles. Un test effectué avec des yoghourts thermisés ne montre le développement d'aucun germe. On a donc utilisé du yoghourt brassé, à $20 \mathrm{~g} / \mathrm{l}$ de matières grasses, composant $35 \mathrm{p}$. $100 \mathrm{du}$ régime. Ce yoghourt était soit vivant, soit thermisé c'est-à-dire soumis $1 \mathrm{mn}$. à une température de $65^{\circ} \mathrm{C}$.

Après 10 ef 13 semaines de régime, des lots de 10 souris de chacun des trois groupes reçoivent par voie sous-cutanée, scit $0,25 \mathrm{ml}$ d'eau isotonique $(\mathrm{NaCl} 9 \mathrm{p} .1000)$, soit $0,25 \mathrm{ml}$ de vaccin antitétanique (IPAD de l'Institut Pasteur à $30 \mathrm{U} / 0,5 \mathrm{ml}$ ) pur ou dilué au $1 / 3$, au $1 / 9$ ou au $1 / 27$. Les animaux sont sacrifiés par décapitation une semaine après la seconde injection. 
Les taux d'immunoglobulines sériques ont été mesurés par immunodiffusion radiale selon Mancini, adaptée par Fayey et Kelvey (1965) sur plaques de gélose. Ce test a été fait sur les sérums individuels des souris témoins ou immunisées avec le vaccin pur. Le restant des sérums a été réuni dans chaque groupe en tenant compte des dilutions de vaccin pour mesurer les taux des antitoxines tétaniques selon la méthode d'Ehrlich adaptée à la souris, ef précisée par Relyveld (1977).

On a prélevé et pesé divers organes. On a effectué une étude histologique de la rate et du thymus après coloration au trichrome de Masson-Goldner ou à l'acide péri-iodique de Schiff.

Pour l'analyse statistique, on a utilisé le test de Kramer (1956) au seuil de 5 p. 100 et le test de rang de Kruskal et Wallis (1952).

\section{Résultats.}

Pesées.

Les pesées hebdomadaires de 10 animaux par groupe sont représentées figure 1. Les souris qui ont reçu les régimes à base de yoghourt sont, après 15 semaines, significativement plus lourdes $\left(+11,5\right.$ p. 100) que celles qui ont reçu le régime témoin $T_{3}$; il n'y a pas de différence entre les poids des souris qui ont reçu du yoghourt contenant des germes vivants ou du yoghourt thermisé.

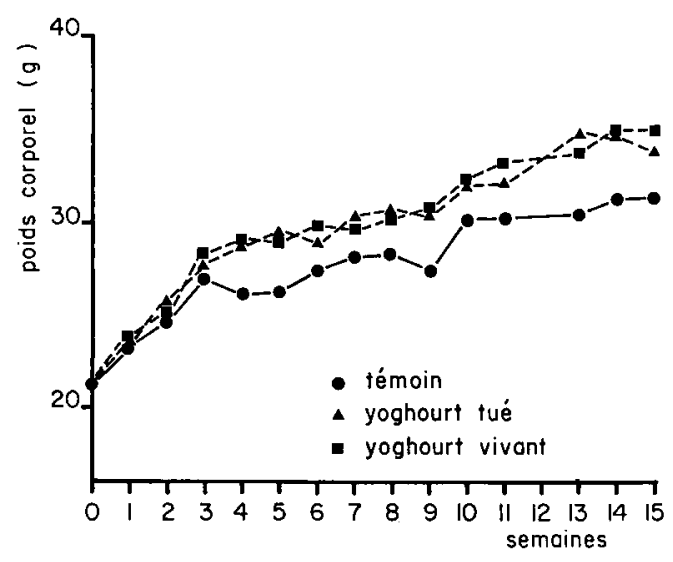

FIG. 1. - Courbe pondérale. Chaque point représente la moyenne de 10 animaux.

Différence significative entre régime témoin et régimes enrichis en yoghourt.

Le poids des rates (tabl. 2) est augmenté de façon significative par le régime enrichi en yoghourt tué, et non significative avec le yoghourt vivant; il est augmenté par la vaccination chez les animaux témoins et chez ceux qui ont reçu le régime enrichi en yoghourt vivant. Le poids des thymus est augmenté chez les souris non vaccinées qui ont reçu du yoghourt thermisé. Cette augmentation ne se retrouve pas chez les animaux vaccinés. 
Les poids du foie et du cœur (tabl. 3), chez l'animal neuf, sont augmentés par les régimes enrichis en yoghourt, mais la différence n'est pas significative entre les deux types de yoghourt.

Le poids du tissu adipeux génital n'est pas significativement modifié par le régime.

\section{TABLEAU 2}

Poids des rates ef thymus

\begin{tabular}{|c|c|c|c|c|c|}
\hline Régime & $\begin{array}{c}\text { Nombre } \\
\text { de } \\
\text { souris }\end{array}$ & $\begin{array}{c}\text { Vaccin } \\
\text { antitétanique }\end{array}$ & $\begin{array}{c}\text { Poids } \\
\text { corporel } \\
\text { (g) }\end{array}$ & $\begin{array}{l}\text { Rate } \\
\text { (mg) }\end{array}$ & $\begin{array}{c}\text { Thymus } \\
\text { (mg) }\end{array}$ \\
\hline \multirow[t]{2}{*}{$\mathrm{T}_{3} \ldots \ldots \ldots \ldots$} & 10 & - & $\begin{array}{r}31,3 \\
+\quad 1,15\end{array}$ & $\begin{array}{r}146,36 \\
\pm \quad 9,67\end{array}$ & $\begin{array}{r}47,38 \\
\pm \quad 2,67\end{array}$ \\
\hline & 10 & $\dot{t}^{-}$ & $\begin{array}{c}31,5 \\
\pm \quad 0,50\end{array}$ & $\begin{array}{r}171,1\left({ }^{c}\right) \\
\pm \quad 13,6\end{array}$ & $\begin{array}{r}44,08 \\
+\quad 3,40\end{array}$ \\
\hline \multirow{2}{*}{ Yoghourt tué } & 10 & - & 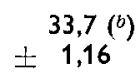 & $\begin{array}{c}177,6\left(^{a}\right) \\
\pm \quad 7,81\end{array}$ & $\begin{array}{r}55,5(a) \\
\pm \quad 2,91\end{array}$ \\
\hline & 10 & + & $\begin{array}{r}36,0 \\
+\quad 1,73\end{array}$ & $\begin{array}{l}177,8\left(^{a}\right) \\
\pm \quad 11,5\end{array}$ & $\begin{array}{r}43,60 \\
\pm \quad 4,20\end{array}$ \\
\hline \multirow{2}{*}{ Yoghourt vivant } & 10 & - & $\begin{array}{r}34,7{ }^{(b)} \\
\pm \quad 1,02\end{array}$ & $\begin{array}{r}157,0 \\
\pm \quad 10,3\end{array}$ & $\begin{array}{r}49,59 \\
+\quad 2,90\end{array}$ \\
\hline & 10 & + & $\begin{array}{r}35,4 \\
+\quad 1,25\end{array}$ & $\begin{array}{c}183,8(a)(c) \\
+\quad 9,00\end{array}$ & $\begin{array}{r}43,50 \\
+\quad 3,74\end{array}$ \\
\hline
\end{tabular}

$\left(^{a}\right)$ Différence significative $(\mathrm{P}<0,05)$ avec le groupe témoin $\mathrm{T}_{3}$.

$(b)$ Différence significative entre l'ensemble des lots yoghourt et le groupe témoins $T_{3}$.

(c) Différence significative entre vaccinés et non vaccinés.

\section{TABLEAU 3}

Poids des foies, cœurs, tissus adipeux génitaux chez l'animal neuf (non vacciné)

\begin{tabular}{|c|c|c|c|c|c|}
\hline Régime & $\begin{array}{c}\text { Nombre } \\
\text { de } \\
\text { souris }\end{array}$ & $\begin{array}{l}\text { Poids } \\
\text { corporel } \\
\text { (g) }\end{array}$ & $\begin{array}{l}\text { Foie } \\
(g)\end{array}$ & $\begin{array}{r}\text { Cour } \\
\text { (mg) }\end{array}$ & $\begin{array}{c}\text { Tissu adipeux } \\
(\mathrm{mg})\end{array}$ \\
\hline $\mathrm{T}_{3} \ldots \ldots \ldots \ldots \ldots$ & 10 & $\begin{array}{r}31,3 \\
\pm 1,15\end{array}$ & $\begin{array}{r}1,798 \\
\pm 0,084\end{array}$ & $\begin{array}{l}141,2 \\
\pm 3,64\end{array}$ & $\begin{array}{r}517,3 \\
\pm \quad 76,0\end{array}$ \\
\hline Yoghourt tué $\ldots . .$. & 10 & & & $\begin{array}{l}168,1\left(^{(a)}\right. \\
\pm 4,27\end{array}$ & $\begin{array}{r}522,8 \\
+\quad 87,4\end{array}$ \\
\hline Yoghourt vivant .... & 10 & $\begin{aligned} & 34,7{ }^{(b)} \\
\pm & 1,02\end{aligned}$ & $\begin{array}{r}1,950 \\
\pm 0,059\end{array}$ & $\begin{array}{l}163,7\left({ }^{a}\right) \\
\pm 5,19\end{array}$ & $\begin{array}{r}732,9 \\
+131,4\end{array}$ \\
\hline
\end{tabular}

${ }^{(a)}$ Différence significative $(\mathrm{P}<0,05)$ avec le groupe témoin $\mathrm{T}_{3}$.

(b) Différence significative entre l'ensemble des lots yohourt et le groupe témoin $\mathrm{T}_{3}$. 
Histologie.

Rates. - A l'histologie, chez les animaux vaccinés, les rates des souris ayant reçu du yoghourt vivant sont hyperactives : on observe une turgescence massive des follicules lymphoïdes. Les espaces $T$ périartériolaires sont très développés. Le réticulum contient de nombreux lymphocytes.

Chez les souris ayant reçu du yoghourt tué, on observe une diminution d'activité par rapport aux précédentes. Cette diminution porte sur les follicules lymphoïdes qui sont moins turgescents, et sur les espaces $T$ qui sont moins développés. Le réticulum présente autant d'éléments lymphoïdes que dans le cas précédent.

Chez les souris au régime témoin $\mathrm{T}_{3}$, les rates sont beaucoup moins réactionnelles que dans le cas précédent. On ne constate en effet pas d'hyperplasie des follicules (sauf chez un animal), à la différence des cas précédents. Dans le réticulum, on observe autant d'éléments lymphoïdes que dans les autres groupes.

- Chez les animaux non vaccinés, les rates des souris ayant reçu du yoghourt vivant sont moins actives que dans le groupe de référence vacciné : hyperplasie modérée des follicules ef éléments lymphoïdes moins nombreux que chez les vaccinés.

Chez les souris ayant reçu du yoghourt tué, les rates sont un peu moins actives que dans le cas précédent, et on observe une diminution nette d'activité par rapport aux animaux du groupe de référence vacciné.

Chez les souris mises au régime témoin $T_{3}$, les rates sont normales, c'est-à-dire moins actives qu'avec les régimes enrichis en yoghourt.

On a effectué une estimation grossière du développement des follicules lymphoïdes dans les rates. Pour cela, on a choisi une coupe histologique dans une zone paraéquatoriale du follicule, dans la rate de chaque animal. Cette coupe a été projetée sur le verre dépoli d'un microscope (Projectina-Altstaetten SG, Suisse). Après avoir dessiné les contours des follicules sur un papier calque posé sur le verre dépoli, la surface du papier leur correspondant a été découpée et pesée. On retrouve la même progression entre les animaux soumis aux différents régimes, sans cependant qu'apparaisse de différence entre vaccinés et non vaccinés (tabl. 4).

TABLEAU 4

Tissu lymphoïde splénique

\begin{tabular}{lccc}
\hline Régime & $\begin{array}{c}\text { Vaccin } \\
\text { antitétanique }\end{array}$ & $\begin{array}{c}\text { Nombre } \\
\text { de souris }\end{array}$ & $\begin{array}{c}\text { Follicule lymphoïde } \\
(\mathrm{mg})\end{array}$ \\
\hline$T_{3} \ldots \ldots \ldots \ldots \ldots \ldots \ldots$ & - & 3 & $22,43 \pm 0,50$ \\
\hline Yoghourt tué......... & + & 4 & $24,4 \pm 3,88$ \\
\hline Yoghourt vivant $\ldots \ldots \ldots$ & - & 5 & $28,4 \pm 4,53$ \\
& - & 5 & $30,2 \pm 3,10$ \\
\hline
\end{tabular}

Le poids des follicules lymphoïdes représente la moyenne des animaux du groupe : il s'agit du poids du papier sur lequel a été relevée la surface du follicule sur une coupe de tissu splénique, projetée sur un écran intégré au microscope.

Les souris vaccinés ont reçu $0,25 \mathrm{ml}$ de vaccin non dilué.

(a) Différence significative entre les deux groupes correspondants. 
Thymus. - L'examen histologique du thymus, chez les animaux vaccinés, met en évidence chez les souris ayant reçu du yoghourt vivant une grande richesse en éléments lymphoïdes. On constate la pénétration de très nombreux lymphocytes de la corticale vers la médullaire, ce qui témoigne d'une stimulation. On observe la présence de nombreux petits lymphocytes (thymocytes).

Chez les souris ayant reçu du yoghourt tué, le thymus est encore actif avec hyperplasie lymphoïde, mais moins dynamique que dans le cas précédent : le passage des lymphocytes de la corticale vers la médullaire est plus réduit. Il s'agit toujours d'une stimulation de niveau élevé, mais moins qu'avec le yoghourt vivant.

Chez les souris soumises au régime témoin $\mathrm{T}_{3}$, le thymus paraît plus petit que dans les cas précédents. On observe peu de différence par rapport aux animaux ayant reçu du yoghourt.

- Chez les animaux non vaccinés, les souris ayant reçu du yoghourt vivant ont un thymus moins stimulé que celui des vaccinées.

Chez les souris ayant reçu du yoghourt tué, l'activité du thymus est diminuée par rapport au cas précédent, et bien sûr par rapport aux souris vaccinées mises au même régime.

Chez les souris mises au régime témoin $\mathrm{T}_{3}$, le thymus présente l'aspect d'un organe au repos, et on note une régression adipeuse partielle.

En conclusion, les réactions sont plus faciles à voir au niveau de la rate que du thymus. D'autre part, l'adjonction des bacilles vivants du yoghourt, tant pour les animaux vaccinés que non vaccinés, provoque une hyperplasie, notamment de la rate, plus forte que celle visible avec les bacilles tués. La vaccination renforce cetfe différence.

\section{- Immunoglobulines.}

Le taux des immunoglobulines sériques figure dans le tableau 5 . On voit que les taux des $\lg _{2}$ b et des $\lg M$ ne sont pas modifiés par le régime alimentaire ou la vaccination.

\section{TABLEAU 5}

Toux d'immunoglobulines sériques $(\mathrm{mg} / \mathrm{dl})$

\begin{tabular}{|c|c|c|c|c|c|c|c|}
\hline Régime & $\begin{array}{c}\text { Vaccin } \\
\text { antitétanique }\end{array}$ & $\lg G_{1}$ & & $\lg G_{2} a$ & $\lg G_{2} b$ & $\lg A$ & $\lg M$ \\
\hline $\begin{array}{l}T_{3} \ldots \ldots \ldots \ldots \ldots \\
\text { Yoghourt tué ....... } \\
\text { Yoghourt vivant.... }\end{array}$ & $(-)$ & $\begin{array}{l}115 \pm 18,3 \\
179 \pm 49,2 \\
111 \pm 13,4\end{array}$ & $\begin{array}{l}207 \\
174 \\
529\end{array}$ & $\begin{array}{l} \pm 70,3 \\
\pm 72,9 \\
\pm 44,0\left({ }^{a}\right)\left({ }^{b}\right)\end{array}$ & $\begin{array}{l}60 \pm 7,2 \\
66 \pm 6,2 \\
60 \pm 8,6\end{array}$ & $\begin{array}{l}\text { traces } \\
\text { traces } \\
\text { traces }\end{array}$ & $\begin{array}{l}34 \pm 5,6 \\
52 \pm 7,6 \\
35 \pm 5,0\end{array}$ \\
\hline $\begin{array}{l}\mathrm{T}_{3} \ldots \ldots \ldots \ldots \ldots \\
\text { Yoghourt tué ....... } \\
\text { Yoghourt vivant.... }\end{array}$ & $(+)$ & $\begin{array}{l}409 \pm 32,5(c) \\
447 \pm 50,8(c) \\
423 \pm 26,3(c)\end{array}$ & $\begin{array}{l}147 \\
315 \\
419,2\end{array}$ & $\begin{array}{l} \pm 59,4 \\
\pm 86,1 \\
\pm 80,6\left({ }^{a}\right)\end{array}$ & $\begin{array}{l}64 \pm 10,0 \\
69 \pm 8,7 \\
87 \pm 17,3\end{array}$ & $\begin{array}{l}30 \pm 8,1 \\
28 \pm 4,3 \\
22 \pm 4,0\end{array}$ & $\begin{array}{l}45 \pm 6,5 \\
51 \pm 9,2 \\
53 \pm 7,6\end{array}$ \\
\hline
\end{tabular}

Les chiffres correspondent à une moyenne de 10 souris par groupe sauf pour les $\lg A$ (5 à 9 souris par lot)

(a) Différence significative avec le lot témoin $T_{3}(p<0,05)$.

(b) Différence significative entre les deux lots de yoghourt.

(c) Différence significative entre les lots vaccinés et non vaccinés. 
Les taux des $\operatorname{lgG}_{1}$ sonf fortement accrus par la vaccination, sans qu'on note d'effet du régime.

Dans le cas des $\lg G_{2} a$, chez les animaux neufs, le faux est multiplié par plus de 2,2 chez les souris ayant absorbé du yoghourt vivant par rapport aux témoins. Le yoghourt tué est sans effet. La vaccination ne modifie pas les taux.

La teneur du sérum en IgA n'est mesurable qu'après vaccination, sans qu'on n'observe d'effet du régime.

- Antitoxines tétaniques.

La figure 2 montre que l'immunité conférée par le vaccin antitétanique est très forte : 25 à $80 \cup$ selon les doses vaccinales. Le pic de la réponse obtenu avec le vaccin dilué à $1 / 3$ est le plus élevé chez les souris qui ont reçu du yoghourt vivant. A la dose la plus faible, les souris témoins ont la réponse la plus basse.

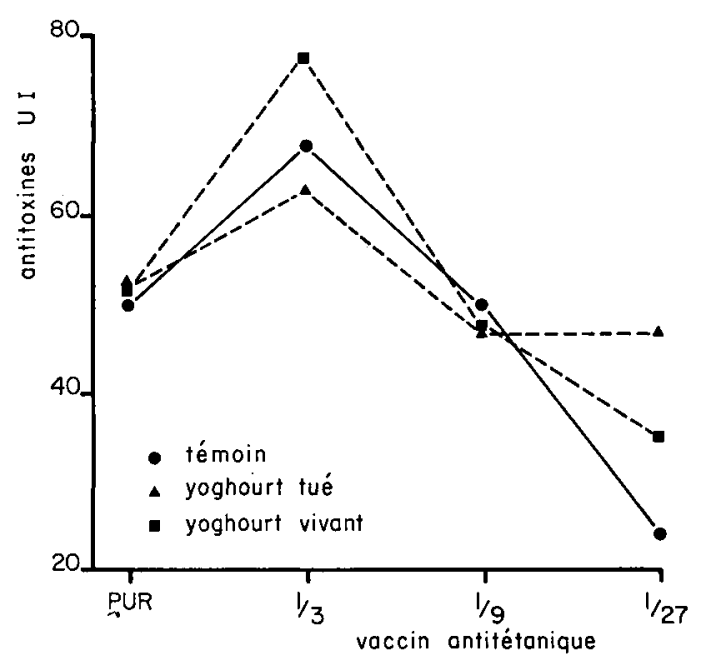

FIG. 2. - Titrage des anitoxines téfaniques. Chaque point représente le taux d'un pool de 10 sérums.

\section{Discussion.}

Les croissances obtenues avec les régimes contenant du yoghourt sont légèrement supérieures à celles observées avec le lot témoin. Ceci a déjà éfé observé chez le rat (Hargrove et Alford, 1978). Les poids des divers organes et tissus (tissu adipeux, cœur, foie, ...) ne diffèrent pas entre eux lorsqu'on tient compte du poids corporel. II semble donc que l'ingestion de yoghourt ait un effet global favorable sur la croissance. II n'esí pas dô aux seuls germes vivants puisqu'il apparaît aussi élevé avec le yoghourł tué. On ne peut dire s'il est lié à l'effet de la masse bacillaire ou au supplément lacté que représente cet apport à la ration témoin des animaux. Il ne peut s'agir de l'action d'un supplément de vitamines apporté par le yoghourt incorporé au régime car le taux de vitamines n'y est pas plus élevé que dans le régime témoin.

En ce qui concerne le taux des diverses immunoglobulines, Molinari, Ebersole ef 
Platt (1974) le trouvent double du nôtre : ceci peut être attribué au fait qu'ils ont travaillé sur une autre lignée de souris, ef dans des conditions d'environnement qui peuvent être différentes, mais leur technique est très proche de la nôtre.

Les régimes comme la vaccination ont des effets manifestes sur la réponse lgG. En effet, globalement, chez les animaux neufs la classe des IgG est multipliée par 2 chez ceux qui ont reçu le régime au yoghourt vivant par rapport aux témoins. La vaccination augmente de 63 p. 100 ce taux chez les témoins. II est double chez ceux qui ont reçu le régime thermisé. En revanche, ils ne sont que légèrement plus élevés chez les animaux qui ont reçu du yoghourt vivant ( +32 p. 100). Le vaccin n'apporte pas d'augmentation du taux lorsque les niveaux des IgG étaient déjà accrus par le régime. L'augmentation de la réponse lgG porte principalement sur la sous-classe des $\lg _{2}$ a dans le cas des animaux neufs et sur la sous-classe des $\lg _{1}$ après vaccination.

Molinari ef al. (1974) ne trouvent pas d'augmentation du taux sérique des diverses immunoglobulines après immunisation par voie orale de souris conventionnelles ou axéniques avec $E$. coli ou $C$. albicans tués. Il en est de même pour nos résultałs avec le yoghourt tué chez les souris non vaccinées. Benveniste et al. (1971), avec une technique moins quantitative que celle que nous avons utilisée, ont enregistré une augmentation du taux des $\lg G_{2}$ ef $\lg G_{1}$ sériques après immunisation et contamination de souris axéniques ou holoxéniques par divers antigènes microbiens. Bazin, André et Heremans (1973) ont aussi signalé une élévation du taux des immunoglobulines sériques après implantation par voie orale d'antigènes vivants ou non chez des animaux axéniques ou conventionnels : ces deux derniers résultats sont conformes aux nôtres, avec les germes vivants du yoghourt donné avant vaccination pour les $\lg _{2} a$, et pour les $\lg _{1}$ après vaccination, quel que soit le régime. Chez les souris vaccinées recevant du yoghourt tué, l'augmentation du taux des $\lg _{2}$ a n'est pas significative. Avec l'administration de yoghourt vivant, le taux des $\lg _{2}$ a double chez les animaux : ceci nous paraît le résultat le plus marquant. Ajoutons que Molinari n'a pas trouvé d'augmentation du taux des anticorps sériques vis-à-vis de Candida ou de E. coli après administration de ces germes tués ; il ne nous a pas encore été possible de titrer les anticorps visà-vis des bacilles du yoghourt.

Le taux des IgA dans nos expériences est très faible, avant vaccination, par cette technique dans le sérum des trois groupes d'animaux. La vaccination élève ce taux. Benveniste et al. (1971) avaient observé des taux d'lgA faibles, mais détectables chez des souris conventionnelles dès l'âge de 3 semaines, ef à 2 mois, des taux comparables aux animaux adultes. A l'âge où nous avons sacrifié les souris, celles-ci étaient donc capables de synthétiser les IgA. Ces auteurs ont aussi noté une élévation du taux des IgA sériques après immunisation. Cette élévation peut être d'origine sécrétoire ou sérique. On sait que le passage vers le sérum des $\operatorname{lgA}$ instestinales est lent, notamment avec des bacilles non vivants.

Moreau et al. (1978) ont examiné l'effet sur le développement des IgA intestinales, au niveau des plasmocytes, de l'ingestion de souches bactériennes intestinales, vivantes ou tuées, qu'elles proviennent de souris ou d'autres espèces; les bacilles lactiques vivants, bien que capables de s'implanter, sont peu immunogènes. Cependant, ils ont constaté une augmentation de la stimulation si on associe diverses souches bactériennes (ce qui est le cas du yoghourt) ; des bacilles tués (toutefois cette expérience ne s'adressait pas à des bacilles lactiques) administrés par voie orale peuvent être aussi 
immunogènes que des bacilles vivants, à condition que leur concentration soit suffisante.

Quant aux IgM, leur taux demeure assez faible, de même que dans les lignées de souris étudiées par Benveniste et al. (1971). Chez l'animal neuf, on constate une légère augmentation chez les souris recevant des bacilles du yoghourt « tué ». La vaccination n'élève pas significativement le taux des IgM quel que soit le régime. Une élévation s'observe dans les expériences de Benveniste ef al. (1971) qui ont eu recours à d'autres antigènes.

En ce qui concerne le taux d'antitoxines tétaniques, aux concentrations de $1 / 3$ et $1 / 27$ de vaccin, on constate une réponse plus marquée chez les souris soumises au yoghourt vivant. Il pourrait s'agir d'un effet adjuvant du yoghourt vivant. L'administration de vaccin non dilué masque probablement les différences. Quant à la dose la plus faible de vaccin, elle permet de mettre en évidence un effet stimulant du yoghourt tué.

On peut donc dire au niveau de la réponse immunitaire sérique que le régime additionné de yoghourt tué a augmenté chez les souris immunisées le taux des $\lg _{2} a$ ef celui des anticorps antitétaniques avec la dose vaccinale la plus faible.

L'administration de yoghourt vivant entraîne une augmentation marquée du taux des $\lg _{2}$ a chez l'animal neuf, et une augmentation du taux des antitoxines chez l'animal vacciné par une dilution à $1 / 3$ de ce vaccin. On ne peut préciser la part exacte du rôle des bacilles vivants du yoghourt agissant comme immuno-stimulants et celle du supplément nutritionnel apporté par le yoghourt dans cet accroissement de la réponse immunitaire : les anticorps vis-à-vis des bacilles du yoghourt n'ont pu être titrés. Il paraît vraisemblable que les bacilles vivants du yoghourt ont joué un rôle stimulant dans cet accroissement.

L'examen histologique du thymus et surtout de la rate fait apparaître des différences importantes selon le régime administré, chez les animaux neufs comme chez les animaux vaccinés. On observe en effet dans la rate une activité plus grande des follicules lymphoïdes chez les animaux du lot yoghourt vivant que chez les animaux du lot yoghourt thermisé, les différences étant significatives entre ces lots, et par rapport aux animaux soumis au régime témoin $T_{3}$. Ces différences portent à la fois sur les cellules $B$ et T. Mais dans le réticulum, il existe autant d'éléments lymphoïdes chez les animaux vaccinés, quel que soit le régime.

C'est donc en particulier la réponse immunitaire qui est stimulée par le régime contenant du yoghourt, cette réponse étant renforcée chez les animaux vaccinés.

L'action inhibitrice du yoghourt ou d'extraits sur la prolifération de cellules tumorales (Bogdanov et al., 1975) chez la souris (Reddy et al., 1973) peut être due à différents mécanismes (Farmer, Reddy et Shahani, 1974). Nos résultats montrent qu'un effet sur la réponse immunitaire n'est pas à exclure. Nous montrons que l'administration de yoghourt vivant modifie les deux types de réponse immunitaire spécifique. Cet effet est plus marqué lorsque la flore du yoghourt est vivante que lorsqu'elle est tuée. Ceci peut s'expliquer par une possibilité d'implantation et de multiplication de cette flore chez la souris conventionnelle. Ce mécanisme paraît le plus vraisemblable pour expliquer les différences observées entre les yoghourts à flore vivante ou thermisée. La possibilité, chez un hôte à flore non contrôlée, d'une implantation d'une flore apportée par le régime, reste un point controversé (Hargrove et Alford, 1978 ; Moreau et al., 1978). Nous n'avons pas vérifié ce qu'il en était pour nos souris. 


\section{Conclusions.}

L'ingestion sous forme de yoghourt d'une flore composée de bacilles lactiques et de streptocoques thermophiles vivants ou tués par thermisation favorise légèrement la croissance de la souris. Les germes administrés, qu'ils soient vivants ou fués, stimulent l'activité immunitaire de la rate et du thymus. Ces effets sont plus marqués lorsque la flore est vivante. Dans ce cas, on observe une augmentation importante du taux sérique des $\lg _{2} a$. La vaccination antitétanique avec un vaccin dilué à $1 / 3$ et à $1 / 27$ élève le taux des $\lg _{1}$; le taux des antitoxines avec le vaccin dilué à $1 / 3$ est élevé, en particulier chez la souris recevant du yoghourt vivant; la vaccination stimule la réponse immunitaire de la rate et du thymus à un plus haut niveau que ne le faisaif l'administration de yoghourt chez l'animal neuf.

Reçu en en juin 1979.

Accepté en décembre 1979.

Remerciements. - Nous remercions Monsieur Hurtrel (Société Gervais-Danone) pour les comptages de la flore des yoghourts mélangés au régime de la souris, ainsi que Messieurs A. Alexiu et J. P. Suquet pour leurs assistance technique. - Ce travail a été réalisé avec l'aide d'un contrat de la Fondation Française de Nutrition.

\section{Références}

BAZIN H., ANDRE C., HEREMANS J. F., 1973. Réponses immunologiques induites par voie orale. Ann. Immunol., 124C, 253-272.

BENVENISTE J., LESPINATS G., ADAM C., SALOMON J. C., 1971. Immunoglobulins in intact, immunized, and contaminated axenic mice : study of serum IgA. J. Immunol., 107, 1647-1654.

BENVENISTE J., LESPINATS G., SALOMON J. C., 1971. Serum and secretory IgA in axenic and holoxenic mice. J. Immunol., 107, 1656-1662.

BOGDANOV I. G., DALEV P. G., GUREVICH A. I., KOLOSOV M. N., MALKOVA V. P., PLEMYANNIKOVA L. A., SOROKINA I. B., 1975. Antitumor glycopeptides from lactobacillus bulgaricus cell wall. FEBS Leffers, 57, 259.

FARMER R. E., REDDY G. V., SHAHANI K. M., 1974. Antitumor activity of yoghurt fractions. J. Dairy Sci., 57, 582-583.

FAHEY J. L., MCKELVEY E. M., 1965. Quantitative determination of serum immunoglobulins in antibody agar plates. J. Immunol., 94, 84-90.

HARGROVE R., ALFORD J., 1978. Growth rate and feed efficiency of rats fed yogurt and other fermented milks. J. Dairy Sci., 61, 11-19.

KRAMER C. Y., 1956. Extension of multiple range tests to group means with inequal numbers of replications. Biometrics, 12, 307-310.

KRUSKAL W. H., WALLIS W. A., 1952. Use of ranks in one-criferion variance analysis. J. amer. Statist. Ass., 47, 583-621.

LEMONNIER D., 1972. Effect of age, sex and site on the cellularity of the adipose tissue in mice and rats rendered obese by a high fat diet. J. clin. Invest., 51, 2907-2915.

MANCINI G., CARBONARA A. O., HEREMANS J., 1965. Immunochemical quantitation of antigens by single radial immunodiffusion. Immunochemistry, 2, 235-254.

MOLINARI J. A., EBERSOLE J. L., PLATT D., 1974. Investigation of secretory immunoglobulins in saliva from germ-free mice. Infect. Immun., 10, 1207-1212.

MOREAU M. C., DUCLUZEAU R., GUY-GRAND D., MULLER M. C., 1978. Increase in the population of duodenal immunoglobulin A plasmocytes in axenic mice associated with different living or dead bacterial strains of intestinal origin. Infect. Immun., 21, 532-539.

REDDY G. V., SHAHANI K., BANERJEE M., 1973. Inhibitory effect of yogurt on Ehrlich ascites tumorcell proliferation. J. nat Cancer Inst., 50, 815-817.

RELYVELD E., 1977. Titrage in vitro des anticorps antidiphtériques et antitétaniques à plusieurs niveaux. J. biol. Standard., 5, 45-55. 